\title{
Gravitational Waveguides in Cosmology
}

\author{
S. Capozziello ${ }^{1,2}$, R. de Ritis ${ }^{1,2}$, V. Mank'o ${ }^{3,4}$, A.A. Marino ${ }^{1,2}$, G. Marmo ${ }^{1,2}$ \\ ${ }^{1}$ Dipartimento di Scienze Fisiche, Università di Napoli, \\ ${ }^{2}$ Istituto Nazionale di Fisica Nucleare, Sezione di Napoli, \\ Mostra d'Oltremare pad. 19 I-80125 Napoli, Italy, \\ ${ }^{3}$ Osservatorio Astronomico di Capodimonte, \\ Via Moiariello 16 I-80131 Napoli, Italy \\ ${ }^{4}$ Lebedev Physical Institute, Leninsky Pr., 53, Moscow 117924, Russia.
}

\begin{abstract}
We discuss the possibility that, besides the usual gravitational lensing, there may exist a sort of gravitational waveguiding in cosmology which could explain some anomalous phenomena which cannot be understood by the current gravitational lensing models as the existence of "brothers" objects having different brilliancy but similar spectra and redshifts posed on the sky with large angular distance. Furthermore, such a phenomena could explain the huge luminosities coming from quasars using the cosmological structures as selfoc-type or planar waveguide. We describe the gravitational waveguide theory and then we discuss possible realizations in cosmology.
\end{abstract}

PACS: 98.80. Dr; 95.30. Sf 


\section{Introduction}

Gravitational lensing has recently become a fundamental tool to investigate large scale structure of the universe and to test cosmological models [1]. One of the most interesting characteristics of gravitational lensing is that it acts on all scales. In fact, it provides a great amount of cosmological and astrophysical applications like the determination of the Hubble parameter $H_{0}$ via the measurement of time delay $\Delta t$ between the observed lightcurves of multiply imaged extragalactic sources [2], [3]; the possibility of weighing the mass and describing the potential of lensing galaxies and galaxy clusters from the observation of multiply imaged quasars, arcs and arclets [4], [5]. Furthermore, the gravitational lensing plays a leading role in searching for dark matter since the frequency of multiply imaged sources (e.g. quasars) depends on the cosmological density parameter $\Omega_{L}$ of compact objects. In fact a way to detect compact objects in the universe with masses in the range $10^{-5} M_{\odot}-100 M_{\odot}$ is based upon the detection of lensing effects which produce characteristic light variations of distant compact sources. Particularly promising are the multiply macro-imaged quasars whose lensing galaxy should have a large optical depth for lensing effects [6], [7] [10] (at least 20 objects are identified with definite action of gravitational lensing; see, for example, [11], [12], [13]).

The above kinds of analysis are possible if we have a model explaining the way of forming images such as the above-mentioned arcs, rings or simply double images and predicting the effects of the deflector [8], [9].

From a theoretical point of view, lensing must be treated studying the geometry of the system source-lens-observer. This study is simple if we suppose that these are three points on a plane as well as if we consider thin lens approximation: such hypotheses are reasonable because of the large distances considered. A theoretical model can be worked out by giving a specified form to the lens density, i.e. fixing its structure. From the density function, using the equations derived from the geometry, we can have predictions for the observed deviation of the source light and magnitude of every image. A fundamental issue is how gravitational lensing effects are connected with a theory of large scale structure formation; in other words, how a theory of galaxy formation can directly furnish gravitational lensing models.

It is well known that the gravitational lensing may be explained using the action of a gravitational field on the electromagnetic waves. In this case, the action of a media with corresponding refraction index is, for weak field approximation, completely determined by the Newtonian gravitational potential which deflects and focuses the light rays.

In optics, however, there exist other types of devices, like optical fibers and waveguides which use the same deflection phenomena. The analogy with the action of a gravitational field onto light rays may be extended to incorporate these other structures on the light. Shortly, it is possible to suppose the existence of a sort of gravitational waveguiding [15], [16], [17]. But this remark has not been studied, till now, neither theoretically nor experimentally. On the other hand, structures like cosmic strings, texture and domain walls, which are produced at phase transiton in inflationary models, can evolve into 
today observed filaments, clusters and groups of galaxies and behave in a variety of ways with respect to gravitational lensing effects. In fact, the lensing by cosmic string was suggested as explaination of the observation [18 of twins objects with anomally large angular distance between the partners [19], 20].

The aim of this work is to discuss the properties of possible waveguides in the universe and to suggest the explaination of some phenomena, like quasar huge luminosities and large angular distances between twins, as a by-product of their existence. For example, a filament of galaxies can be considered a sort of waveguide preserving total luminosity of a source, if we have locally an effective gravitational potential of the form $\Phi(\mathbf{r}) \sim \mathbf{r}^{2}$, while the planar structures generated by the motion of cosmic strings (the so called "wakes") can yield cosmological structures where the total flux of light is preserved and the brightness of objects at high redshift, whose radiation passes through such structures, appears higher to a far observer.

Sect. 2 is devoted to the discussion of the gravitational potential intended as the refraction index of geometrical optics.

In Sect. 3, we costruct the optical waveguide model using the paraxial approximation (the so called Fock-Leontovich approximation [21]). We propose a model in which we consider, instead of a simple gravitational lensing effect, the effect of a sort of system of lenses which, combined in files or in planes, results as a waveguide. In Sect. 4, we discuss the eventual cosmological realization of such structures and the connection with observations. Conclusions are drawn in Sect.5.

\section{The propagation of light in a weak gravitational field}

It is possible to discuss the gravitational waveguide properties [15, [16, 17] using the electromagnetic field theory in a gravitational field described by the metric tensor $g_{\mu \nu}$ [11, [13]. In this context, the behaviour of the electromagnetic field without sources in the presence of a gravitational field may be described by the Maxwell equations [28], 29]

$$
\begin{aligned}
& \frac{\partial F_{\alpha \beta}}{\partial x^{\gamma}}+\frac{\partial F_{\beta \gamma}}{\partial x^{\alpha}}+\frac{\partial F_{\gamma \alpha}}{\partial x^{\beta}}=0 ; \\
& \frac{1}{\sqrt{-g}} \frac{\partial}{\partial x^{\beta}}\left(\sqrt{-g} F^{\alpha \beta}\right)=0,
\end{aligned}
$$

where $F^{\alpha \beta}$ is the electromagnetic field tensor and $g$ is the determinant of the fourdimensional metric tensor. For a static gravitational field, these equations can be reduced to the usual Maxwell equations describing the electromagnetic field in media where the dielectric and magnetic tensor permeabilities are connected with the metric tensor $g_{\mu \nu}$ by the equation [27]

$$
\varepsilon_{i k}=\mu_{i k}=-g_{00}^{-1 / 2}\left[\operatorname{det} g_{i k}\right]^{-1 / 2} g_{i k} ; \quad i, k=1,2,3 .
$$


If one has an isotropic model, the metric tensor is diagonal and the refraction index of "media" may be introduced by mimicking by the gravitational field

$$
n(\mathbf{r})=(\varepsilon \mu)^{1 / 2},
$$

(it is worthwhile to note that such a situation can be easily reproduced in cosmology |13]).

Following the standard procedure for deriving the scalar Helmholtz equation [13 for the components of the electromagntetic field from the first order Maxwell equations, we get (for some arbitrary monochromatic component of the electric field)

$$
\frac{\partial^{2} E}{\partial z^{2}}+\frac{\partial^{2} E}{\partial x^{2}}+\frac{\partial^{2} E}{\partial y^{2}}+k^{2} n^{2}(\mathbf{r}) E=0
$$

where $k$ is the wave number. This procedure works if the relative change of diffraction index on distances of the light wavelength is small.

The coordinate $z$, in Eq.(5), is considered as the longitudinal one and it can measure the space distance along the gravitational field structure produced by a mass distribution

with an optical axis. Such a coordinate may also correspond to a distance along the light path inside a planar gravitational field structure produced by a planar matter-energy distribution in some regions of the universe (like a string wake or a wall of galaxies see the discussion in Sect. 4). For weak gravitational fields, considered also to describe usual gravitational lensing effects, the metric tensor components are expressed in terms of the Newton gravitational potential $\Phi$ as [1], [13, 27]

$$
g_{00} \simeq 1+2 \frac{\Phi(\mathbf{r})}{c^{2}} ; \quad g_{i k} \simeq-\delta_{i k}\left(1-2 \frac{\Phi(\mathbf{r})}{c^{2}}\right) ;
$$

where we are assuming the approximation $\Phi / c^{2} \ll 1$. Then, due to relations (3) and (6), the refraction index $n(\mathbf{r})$ in (四) can be expressed in terms of the gravitational potential $\Phi(\mathbf{r})$ produced by some matter distribution. Such a weak field situation is realized for cosmological structures which give rise to the gravitational lensing effects connected to several observable phenomena (multiple images, magnification, image distorsion, arcs and arclets); with some cautions, we can use the same scheme also in strong lensing approximation [13]. Here, we are interested to a specific application which, we believe, is realized by some kinds of gravitational systems: is it possible to realize cosmological waveguide effects considering string-like or planar-like distribution of matter? Are such effects observable? In the next section we construct a gravitational waveguide model using the above hypotheses on gravitational field and light propagation. After we discuss some practical cosmological applications.

\section{The gravitational waveguide model}

If one has a matter distribution with some axis like a cylinder with dust or like a planar slab with dust, it is possible to consider the electromagnetic field radiation propagating 
paraxially. The parabolic approximation [21] is used for describing light propagation in media and in devices as optical fibers [14]. Below, we will discuss the possibility to use this approximation for describing electromagnetic radiation propagating in a weak gravitational field.

Let us consider, the scalar equation (5) and the electric field $E$ of the form

$$
E=n_{0}^{-1 / 2} \Psi \exp \left(i k \int^{z} n_{0}\left(z^{\prime}\right) d z^{\prime}\right) ; \quad n_{0} \equiv n(0,0, z)
$$

where $\Psi(x, y, z)$ is a slowly varying spatial amplitude along the $z$ axis, and exp $(i k n z)$ is a rapidly oscillating phase factor. Its clear that the beam propagation is along the $z$ axis. We rewrite Eq.(5) neglecting second order derivative in longitudinal coordinate $z$ and obtain a Schrödinger-like equation for $\Psi$ :

$$
i \lambda \frac{\partial \Psi}{\partial \xi}=-\frac{\lambda^{2}}{2}\left(\frac{\partial^{2} \Psi}{\partial x^{2}}+\frac{\partial^{2} \Psi}{\partial y^{2}}\right)+\frac{1}{2}\left[n_{0}^{2}(z)-n^{2}(x, y, z)\right] \Psi
$$

where $\lambda$ is the electromagnetic radiation wavelength and we adopt the new variable

$$
\xi=\int^{z} \frac{d z^{\prime}}{n_{0}\left(z^{\prime}\right)}
$$

normalized with respect to the refraction index [15] (for our application, $n_{0}(z) \simeq 1$ so that $\xi$ coincides essentially with $z$ ).

At this point, it is worthwhile to note that if one has the distribution of the matter in the form of cylinder with a constant (dust) density $\rho_{0}$, the gravitational potential inside has a parabolic profile providing waveguide effect for electromagnetic radiation analogous to sel-foc optical waveguides realized in fiber optics. In this case, Schrödingerlike equation is that of two-dimensional quantum harmonic oscillator for which the mode solutions exist in the form of Gauss-Hermite polynomials (see, for example, [22]). In the case of inhomogeneous longitudinal dust distribution in the cylinder (that is $\rho(z)$ ), the Schrödinger-like equation describes the model of two-dimensional parametric oscillator for which the mode solutions, in the form of modified Gaussian and Gauss-Hermite polynomials, exist with parameters determined by the density dependence on longitudinal coordinate.

As a side remark, it is interesting to stress that, considering again Eq.(8), the term in square brackets in the rhs plays the role of the potential in a usual Schrödinger equation; the role of Planck constant is now assumed by $\lambda$. Since the refraction index can be expressed in terms of the Newtonian potential when we consider the propagation of light in a gravitational field, we can write the potential in (8) as

$$
U(\mathbf{r})=\frac{2}{c^{2}}[\Phi(x, y, z)-\Phi(0,0, z)] .
$$

The waveguide effect depends specifically on the shape of potential (10): for example, the radiation from a remote source does not attenuate if $U \sim r^{2}$; this situation is realized 
supposing a "filamentary" or a "planar" mass distribution with constant density $\rho$. Due to the Poisson equation, the potential inside the filament is a quadratic function of the transverse coordinates, that is of $r=\sqrt{x^{2}+y^{2}}$ in the case of the filament and of $r=x$ in the case of the planar structure (obviously the light propagates in the "remaining" coordinates: $z$ for the filament, $z, y$ for the plane). In other words, if the radiation, travelling from some source, undergoes a waveguide effect, it does not attenuate like $1 / R^{2}$ as usual, but it is, in some sense conserved; this fact means that the source brightness will turn out to be much stronger than the brightness of analogous objects located at the same distance (i.e. at the same redshift $Z$ ) and the apparent energy released by the source will be anomalously large.

To fix the ideas, let us estimate how the electric field (7) propagates into an ideal filament whose internal potential is

$$
U(r)=\frac{1}{2} \omega^{2} r^{2}, \quad \omega^{2}=\frac{4 \pi G \rho}{c^{2}}
$$

where $\rho$ is constant and $G$ is the Newton constant. A spherical wave from a source,

$$
E=(1 / R) \exp (i k R)
$$

can be represented in the paraxial approximation as

$$
E(z, r)=\frac{1}{z} \exp \left(i k z+\frac{i k r^{2}}{2 z}-\frac{r^{2}}{2 z^{2}}\right),
$$

where we are using the expansion

$$
R=\left(z^{2}+r^{2}\right)^{1 / 2} \approx z\left(1+\frac{r^{2}}{2 z^{2}}\right), \quad r \ll z .
$$

It is realistic to assume $n_{0} \simeq 1$ so that, from (9), $\xi=z$. Assume now that the starting point of the filament of length $L$ is at a distance $l$ from a source shifted by a distance $a$ from the filament axis in the $x$ direction. The amplitude $\Psi$ of the field $E$, entering the wave guide is

$$
\Psi_{\text {in }}=\frac{1}{l} \exp \left[\frac{i k l-1}{2 l^{2}}\left((x-a)^{2}+y^{2}\right)\right]
$$

and so in (12), we have $R=\left(l^{2}+y^{2}+(x-a)^{2}\right)^{1 / 2}$.

We can calculate the amplitude of the field at the exit of the filament by the equation

$$
\Psi_{f}(x, y, l+L)=\int d x_{1} d y_{1} G\left(x, y, l+L, x_{1}, y_{1}, l\right) \Psi_{i n}\left(x_{1}, y_{1}, l\right),
$$

where $G$ is the Green function of Eq.(8). For the potential (11), $G$ has the form

$$
\begin{aligned}
G\left(x, y, l+L, x_{1}, y_{1}, l\right) & =\frac{\omega}{2 \pi i \lambda \sin \omega \lambda} \\
& \times \exp \left(\frac{i \omega\left[\cos \omega L\left(x^{2}+y^{2}+x_{1}^{2}+y_{1}^{2}\right)-2\left(x x_{1}+y y_{1}\right)\right]}{2 \pi i \lambda \sin \omega \lambda}\right)
\end{aligned}
$$


which is the propagator of the harmonic oscillator. The integral (16) is Gaussian and can be exactly evaluated

$$
\begin{aligned}
\Psi_{f} & =\frac{\omega l}{\omega l^{2} \cos \omega L+(l+i \lambda) \sin \omega l} \\
& \times \exp \left(-\frac{1}{2} \frac{\left(x^{2}+y^{2}\right)\left[(\omega l k)^{2}-\omega k(i+k l) \cot \omega l\right]-a^{2} \omega k(i+k l) \cot \omega L}{1-i k l-i k \omega l^{2} \cot \omega l}\right) \\
& \times \exp \left(-\frac{2 x a \omega k(1+k l)}{2 \sin \omega L\left(1-i k l-i k \omega l^{2} \cot \omega L\right)}\right) .
\end{aligned}
$$

The parameter $l$ drops out of the denominator of the pre-exponential factor if the length $L$ satisfies the condition

$$
\tan \omega L=-\omega l .
$$

Eq.(18) is interesting in two limits. If $\omega l \ll 1$, we have

$$
\Psi_{f}=\frac{1}{i \lambda} \exp \left\{-\frac{l+i \lambda}{2 \lambda^{2} l}\left[(x+a)^{2}+y^{2}\right]\right\},
$$

which means that the radiation emerging from a point with coordinate $(a, 0,0)$ is focused near a point with coordinates $(-a, 0, l+L)$ (that is the radius has to be of the order of the wavelength). This means that, when the beam from an extended source is focused inside the waveguide in such a way that, at a distance $L$, Eq.(19) is satisfied, an inverted image of the source is formed, having the very same geometrical dimensions of the source. The waveguide "draws" the source closer to the observer since, if the true distance of the observer from the source is $R$, its image brightness will correspond to that of a similar source at the closer distance

$$
R_{e f f}=R-l-L .
$$

If we do not have $\omega l \ll 1$, we get (neglecting the term $i \lambda / l$ compared with unity)

$$
\Psi_{f}=\frac{\sqrt{1+(\omega l)^{2}}}{i \lambda} \exp \left\{-\frac{1+(\omega l)^{2}}{2 \lambda^{2}}\left[y^{2}+\left(x+\frac{a}{\sqrt{1+(\omega l)^{2}}}\right)^{2}\right]\right\}
$$

from which, in general, the size of the image is decreased by a factor $\sqrt{1+(\omega l)^{1 / 2}}$. The amplitude increases by the same factor, so that the brightness is $\left(R / R_{e f f}\right)$ times larger.

In the opposite limit $\omega l \gg 1$, we have $\tan \omega L \rightarrow \infty$, so that $L \simeq \pi / \omega$, that is the shortest focal length of the waveguide is

$$
L_{f o c}=\sqrt{\frac{\pi c^{2}}{4 G \rho}}
$$

which is the length of focusing of the initial beam of light trapped by the gravitational waveguide. All this arguments apply if the waveguide has (at least roughly) a cylindrical 
geometry. The theory of planar waveguide is similar but we have to consider only $x$ as transverse dimension and not also $y$.

The cosmological feasibility of a waveguide depends on the geometrical dimensions of the structures, on the connected densities and on the limits of applicability of the above idealized scheme. In the next section, we shall discuss these features and the possible candidates which could give rise to observable effects.

\section{Observable effects and cosmic structures as waveguides}

The gravitational waveguide effect has the same physical reason that has the gravitational lenses effect which is the electromagnetic wave deflection by the gravitational field (equivalent to the deflection of light by a refractive media). However, there are essential differences producing specific predictions for observing the waveguide effect. The gravitational lenses are usually considered as compact objects with strong enough gravitational potential. The light rays deflected by gravitational lenses move outside of the matter which forms the gravitational lens itself. The gravitational waveguide as well as optical waveguide is noncompact long structure which may contain small matter density and deflection of the light by each element of the structure is very small. Due to very large scale sizes of the structure (we give an extimation below), the electromagnetic radiation deflection by the gravitational waveguide occurs and, in principle, it may be observed. We will mention, for example, a possibility of brilliancy magnification of the long distanced objects (like quasars) with large red shift as consequence of the waveguiding structure existence between the object and the observer. This effect exists also for a gravitational lens located between the object and the observer, but the long gravitational waveguide may give huge magnification, since the radiation propagates along the waveguide with functional dependence of the intensity on the distance which does not decrease as $\sim 1 / R^{2}$, characteristic for free propagation. The gravitational lens, being a compact object, collects much less light by deflecting the rays to observer than the gravitational waveguide structure transporting to the direction of observer all trapped energy (of course, one needs to take into account losses for scattering and absorbtion). From that point of view, it is possible that enormous amount of radiation emitted by quasars is only seemingly existing. The object may radiate a resonable amount of energy but the existing waveguide structure transmit the energy in high portion to the observer. Similar ideas, related to gravitational lensing, were discussed in [37 but, since above mentioned reasons, the singular lens or even few aligned strong lenses cannot produce effect of many orders of magnitude magnification of brilliancy. The waveguide effect may explain the anomalous high luminosity observed in quasars. In fact, quasars are objects at very high redshift which appear almost as point sources but have luminosity that are about one hundred times than that of a giant elliptical galaxy (quasars have luminosity which range between $10^{38}-10^{41} \mathrm{~W}$ ). For example, PKS 2000-330 has one of the largest known redshifts $(Z=3.78)$ with a luminosity of $10^{40} \mathrm{~W}$. Such a redshift corresponds to a distance of $3700 \mathrm{Mpc}$, if it is assumed that its origin is due to the expansion of the 
universe and the Hubble constant is assumed $H=75 \mathrm{~km} \mathrm{~s}^{-1}$ Mpc. This means that light left the quasar when the size of the universe was one-fifth of its present age where no ordinary galaxies (included the super giant radio-galaxies) are observed. The quasars, often, have both emission and absorption lines in their spectra. The emission spectrum is thought to be produced in the quasar itself; the absorption spectrum, in gas clouds that have either been ejected from the quasar or just happen to lie along the same line of sight. The brightness of quasars may vary rapidly, within a few days or less. Thus, the emitting region can be no larger than a few light-days, i.e. about one hundred astronomic units. This fact excludes that quasars could be galaxies (also if most astronomers think that quasars are extremely active galactic nuclei).

The main question is how to connect this small size with the so high redshift and luminosity. For example, H.C. Arp discovered small systems of quasars and galaxies where some of the components have widely discrepant redshifts [30]. For this reason, quasar high redshift could be produced by some unknown process and not being simply of cosmological origin. This claim is very controversial. However there is a fairly widely accepted preliminary model which, in principle, could unify all the forms of activities in galaxies (Seyfert, radio, Markarian galaxies and BL Lac objects). According to this model, most galaxies contain a compact central nucleus with mass $10^{7}-10^{9} \mathrm{M}_{\odot}$ and diameter $<1$ pc. For some reason, the nucleus may, some times, release an amount of energy exceeding the power of all the rest of the galaxy. If there is only little gas near the nucleus, this leads to a sort of double radio source. If the nucleus contains much gas, the energy is directly released as radiation and one obtains a Seyfert galaxy or, if the luminosity is even larger, a quasar. In fact, the brightest type 1 Seyfert galaxies and faintest quasars are not essentially different in luminosity $\left(\sim 10^{38} \mathrm{~W}\right)$ also if the question of redshift has to be explained (in fact quasar are, apparently, much more distant). Finally, if there is no gas at all near such an active nucleus, one gets BL Lac objects. These objects are similar to quasar but show no emission lines. However the mechanism to release such a large amount of energy from active nuclei or quasars is still unknown. Some people suppose that it is connected to the releasing of gravitational energy due to the interactions of internal components of quasars. This mechanism is extremely more efficient than the releasing of energy during the ordinary nuclear reactions. The necessary gravitational energy could be produced, for example, as consequence of the falling of gas in a very deep potential well as that connected with a very massive black hole. Only with this assumption, it is possible to justify a huge luminosity, a cosmological redshift and a small size for the quasars.

An alternative explaination could come from waveguiding effects. As we have discussed, if light travels within a filamentary or a planar structure, whose Newtonian gravitational potential is quadratic in the transverse coordinates, the radiation is not attenuated, moreover the source brightness is stronger than the brightness of analogous object located at the same distance (that is at the same redshift). In other words, if the light of a quasar undergoes a waveguiding effect, due to some structure along the path between it and us, the apparent energy released by the source will be anomalously large, 
as the object were at a distance (21). Furthermore, if the approximation $\omega l \ll 1$ does not hold, the dimensions of resulting image would be decreased by a factor $\sqrt{1+(\omega l)^{2}}$ while the brightness would be $\left(R / R_{e f f}\right)^{2}$, larger, then explaining how it is possible to obtain so large emission by such (apparently) small objects. In conclusion, the existence of a waveguiding effect may prevents to take into consideration exotic mechanism in order to produce huge amounts of energy (as the existence of a massive black hole inside a galactic core) and it may justify why it is possible to observe so distant objects of small geometrical size.

Another effect concerning the quasars may be directly connected with multiple images in lensing. The waveguide effect does not disappear if the axis of "filament" or if the guide plane is bent smoothly in space. As in the case of gravitational lenses, we can observe "twin" images if part of the radiation comes to the observer directly from the source, and another part is captured by the bent waveguide. The "virtual" image can then turn out to be brighter than the "real" one (in this case we may deal with "brothers" rather than "twins" since parameters like, spectra, emission periods and chemical compositions are similar but the brightnesses are different). Furthermore, such a bending in waveguide could explain large angular separations among the images of the same object which cannot be explained by the current lens models (pointlike lens, thin lens and so on).

Now the issue is: are there cosmic structures which can furnish workable models for waveguides? Have they to be "permanent" structures or may the waveguide effect be accidental (for example an alignment of galaxies of similar density and structure, due to cosmic shear and inhomogeneity, may be available as waveguide just for a limited interval of time [31])? In general both points of view may be reasonable and here we will outline both of them. Furthermore we have to consider the problem of the abundance of such structures: are they common and everywhere in the universe or are they peculiar and located in particular regions (and eras)?

We have to do a first remark on the densities of waveguide structures which allow observable effects [15]. Considering Eq.(23) and introducing into it the critical density of the universe $\rho_{c} \sim 10^{-29} \mathrm{~g} / \mathrm{cm}^{3}$ (that is the value for which the density parameter is $\Omega=1$ ), we obtain $L_{f o c} \sim 5 \times 10^{4} \mathrm{Mpc}$ which is an order of magnitude larger than the observable universe and it is completely unrealistic. On the contrary, considering a typical galactic density as $\rho \sim 10^{-24} \mathrm{~g} / \mathrm{cm}^{3}$, we obtain $L_{f o c} \sim 100 \mathrm{Mpc}$, which is a typical size of large scale structure (e.g. the Great Wall has such dimensions and also a filament of galaxies can have such a length [32]).

However, an important issue has to be taken into consideration: the absorption and the scattering of light by the matter inside the filament or the planar structure increase with density and at certain crital value the waveguide effect can be invalidated [15]. For the smaller frequency of broadcast range (due to strong dependence of the absorbtion cross section on the electromagnetic wavelength) $\sigma \sim \sigma_{T}\left(\omega / \omega_{0}\right)^{4}$, where Thomson crosssection $\sigma_{T}=6 \cdot 10^{-25} \mathrm{~cm}^{2}$ and the characteristic atomic frequency is $\omega_{0} \sim 10^{16} \mathrm{~s}^{-1}$, the ratio $\omega / \omega_{0} \ll 1$, and the absorbtion is small. It means that the absorbtion length $L_{a}=m_{p} / \rho \sigma$, (where the mass of proton $m_{p}$ is approximately equal to the hydrogen 
atom mass) is larger than the focusing length $L_{a}<L_{f o c}$ for the electomagnetic waves of broadcast range. Thus, the magnification of electromagnetic waves may be not masked by essential energy losses due to light absorbtion and scattering processes. However, no restrictions exist practically if the radio band and a thickness of the structure $r>10^{14} \mathrm{~cm}$ are considered.

In such a case, the relative density change between the background and the structure density is valid till $\delta \rho / \rho \leq 1$. This means that we have to stay in a linear perturbation density regime.

By such hypotheses, practically all the observed large scale structures like filaments, walls, bubbles and clusters of galaxies can result as candidates for waveguiding effect if the restrictions on density, potential and waveband are respected (in optical band, such phenomena are possible but the density has to be chosen with some care).

Also primordial structures (produced in inflationary phase transition and surviving later), like cosmic string, could furnish waveguides. In fact, in weak energy limit approximation, such objects are internally described by the Poisson equation $\nabla^{2} \Phi=\rho_{0}$ and externally by $\nabla^{2} \Phi=0$ and, furthermore, they act as gravitational lenses after the formation of the quasar [19, [20]. It is easy to recover an internal potential of the form $\Phi \sim r^{2}$ and, considering the dynamical evolution after the decoupling, lengths in the required ranges for waveguide (e.g. $\sim 100 \mathrm{Mpc}$ ). The main problem is due to the fact that also after the evolution to macroscopic sizes, strings remain "wires" without becoming cylinders, that is their thickness remain well below $r \sim 10^{14} \mathrm{~cm}$, the minimal value required to get observable effects. However, we have not considered the scaling solutions (see for example [33]) from which such wires could evolve in cylindrical structures (with transverse sizes non trivial with respect to the background).

Other two interesting features are connected with cosmic strings: the first is that their motion with respect to the background produces wakes and filaments which, later, are able to evolve in large scale structures systems of galaxies [34]. For example, at decoupling $(Z \sim 1000)$, a string can produce a wake, which consists in a planar structure, with side $\Delta r \sim 1 \mathrm{Mpc}$ and constant surface density $\sigma_{0} \sim 3 \times 10^{11} \mathrm{M}_{\odot} \mathrm{Mpc}^{-2}$. Such a feature is interesting for large scale structure formation and can yield a planar waveguide with today observable effects. The second fact is that inflationary phase transition can produce a large amount of cosmic strings which, evolving, can give rise to a string network pervading all the universe [1],[19]. In such a case, if they evolve in cylindrical or planar structure, we may expect large probabilities to detect waveguiding effects.

Concerning the second point of view (that is the existence of temporary waveguiding effects), it could be related to the observation of objects possessing anomalously large (compared with their neighbours) angular motion velocities (an analysis in this sense could come out in mapping galaxies with respect to their redshift and proper velocities, see for example [36]). Such a phenomenon could mean that one observes not the object itself, but its image transmitted through the moving gravitational waveguide. The waveguide itself could change its form or it could be due to temporary alignments of lens galaxies. In this case, the image of the object could move with essentially different 
angular velocity than that of the observable neighbour objects whose light reaches the observer directly (not throught the waveguide). The discovery of long distanced objects with anomalous velocity (and brightness) could support the hypothesis of gravitational waveguide effect, while the evolution of the waveguide, its destruction or change of the axis direction (from the orientation to the Earth) could produce the effect of the disappearence (or the appearence) of the observed object. For this analysis, it is crucial to consider long period astronomical observations and deep pencil beam surveys of galaxies and quasars.

\section{Conclusions}

In this paper, we have discussed the possible existence of gravitational waveguide effects in the universe and constructed a radiation propagation model to realize them. As in the case of gravitational lensing, several phenomena and cosmic structures could confirm their

existence, starting from primordial object like cosmic strings to temporary alignement of evolved late-type galaxies. Furthermore, due to the wavelength considered, they could give observable effects in optic, radio or microwave bands or, alternatively, considering the propagation of other weak interacting particles as the neutrinos. The experimental feasibility for the detection could have serious troubles due to the need of long period observations or due to the discrimination among data coming from objects which have undergone waveguide effects and objects which not.

In any case, if such a hypothesis will be confirmed in some of the above quoted senses, we shall need a profound revision of our conceptions of large scale structure and matter distribution.

Finally we want to stress that our treatment does not concern only electromagnetic radiation: actually a waveguide effect could be observed also for streams of neutrinos or other particles which gravitationally interact with the filament (or the plane), in this sense it could result useful also in other fields of astrophysics and fundamental physics.

\section{REFERENCES}

1. P.J.E. Peebles Principles of Physical Cosmology (Princeton Univ. Press., Princeton 1993)

2. U. Borgeest Astron. Astrophys. 128162 (1983)

3. J.A. Frieman, D.D. Harari, and G.C. Surpi Phys. Rev. D 504895 (1994)

4. B. Fort, and Y. Mellier Astron. Astrophys. Rev. 5239 (1994)

5. T.J. Broadhurst, A.N. Taylor, and J.A. Peakock Ap. J. 43849 (1995)

6. B. Paczynski Gravitational Lenses Lecture Notes in Physics 406, p. 163, SpringerVerlag, Berlin (1992) 
7. R. Kaiser Gravitational Lenses Lecture Notes in Physics 404, p. 143, SpringerVerlag, Berlin (1992)

8. M. Bartelmann and P. Schneider Astron. Astrophys. 2681 (1993)

9. R. Kormann, P. Schneider, and M. Bartelmann Astron. Astrophys. 284, 285 (1994)

10. D. Walsh, R.F. Carswell, and R.J. Weymann Nat. 279, 381 (1979)

11. P.V. Blioh and A.A. Minakov Gravitational Lenses Kiev, Naukova Dumka (1989) (in Russian)

12. P. Schneider Cosmological Applications of Gravitational Lensing, Lecture Notes in Physics, eds. E. Martinez-Gonzales, J.L. Sanz, Springer Verlag, Berlin (1996), astro-ph/9512047 (1995).

13. P.Schneider, J. Ehlers, and E.E. Falco Gravitational Lenses Springer-Verlag, Berlin (1992).

14. E. M. Lifšits, L.P. Pitaevskij Elettrodinamica dei mezzi continui, Ed. Riuniti, Roma (1986).

15. V.V. Dodonov and V.I. Man'ko, Gravitational waveguide, Preprint of the Lebedev Physical Institute Proceedings, No. 255 (Moscow, 1988); J. Soviet Laser Research (Plenum Press), 10, 240 (1989); Invariants and Evolution of Nonstationary Quantum Systems Proceedings of the Lebedev Physical Institute, 183 Nova Science Publishers N.Y. (1989).

16. V.V. Dodonov in: Proceedings of the First International Sakharov Conference Moskow May 21-25 (1992), p. 241 Edt. L. V. Keldysh, V. Ya. Fainberg, Nova Science Publishers N.Y. (1993)

17. V.V. Dodonov, O.V. Man'ko, and V.I. Man'ko, in Sqeezed and Correlated States of Quantum Systems Proceedings of the Lebedev Physical Institute, 205 p. 217 Nova Science Publishers N.Y. (1993).

18. E. L. Turner, D. P. Schneider, B. F. Burke, J. N. Hewitt, G. L. Langston, J. E. Gunn, C. R. Lawrence, and M. Schmidt, Nature, 321142 (1986).

19. A. Vilenkin, Ap. J. 282, L51 (1984); Phys. Rep. 121, 263 (1985);

20. J.R. Gott III, Ap. J. 288, 422 (1985).

21. A. M. Leontovich and V. A. Fock, Zh. Eksp. Teor. Fiz., 16, 557 (1946).

22. V. I. Man'ko, in Lee Methods in Optics, Lecture Notes in Physics, Eds. S. Mondragon and K.-B. Wolf, 250193 (1986). 
23. E.W. Kolb, M.S. Turner The Early Universe (Addison-Wesley Pub. Co., Menlo Park 1990)

24. J. Binney, S. Tremaine Galactic Dynamics (Princeton Univ. Press.: Princeton 1987)

25. D. Mihalas, J.J. Binney Galactic Astronomy 2nd. ed. San Francisco, Freeman (1981)

26. S. Refsdal, J. Surdej Rep. Prog. Phys. 57, 117 (1994).

27. L. D. Landau and E. M. Lifšits The Classical Theory of Fields, Pergamon Press, N.Y. (1975).

28. G.V. Skrotsky, Doklady AN SSSR, 114, 73 (1957).

29. J. Plebansky, Phys. Rev. , 118, 1396 (1960).

30. H.C. Arp Quasars, Redshifts and Controversies Berkeley: Interstellar Media, $\$ 7$ (1987).

31. F. Valdes, J.A. Tyson, J.F. Jarvis Ap. J. 271, 431 (1983).

32. V. de Lapparent, M.J. Geller, J.P. Huchra Ap. J. 302, L1 (1986).

M.J. Geller, J.P. Huchra Science 246, 897 (1989).

33. T.W.B. Kibble J. Phys. A9, 1387 (1976).

34. T. Vachaspati, A. Vilenkin, Phys. Rev. Lett. 67, 1057 (1991).

35. S. Seitz, P. Schneider, J. Ehlers, Class. Quantum Grav. 11, 23 (1994).

36. M. Davis, J. Huchra, D.W. Latham, J. Tonry Ap. J. 253, 423 (1982)

37. J. M. Barnothy, Astron. J., 70, 666 (1965). 